\title{
Plastic Circuit Reliability and Design for Recycling
}

\author{
David V. Thiel, Madhusudanrao Neeli and Sundra Raj* \\ Centre for Wireless Monitoring and Applications \\ Box 49, N44 Griffith University, Nathan Qld 4111 Australia \\ d.thiel@griffith.edu.au,m.neeli@griffith.edu.au \\ *Flextronics International Singapore \\ 2 Changi South Lane, 486123, Singapore. \\ Sundra.Raj@my.flextronics.com
}

\begin{abstract}
Electronics is a major contributor to solid waste streams around the world. The manufacturing and the recycling processes currently used in the electronics industry are energy intensive and are accompanied by noxious gaseous and liquid waste. Circuits in Plastic (CiP) is a new technology designed to address issues. Circuit components are placed in a plastic substrate, conductive tracks are screen printed and the completed completed by thermally binding a cover sheet of the same material. The circuits are disassembled by mechanical means. While the process requires much less energy and produces minimal waste, the process must be reliable. Mechanical experiments and thermal modeling have supported the viability of the technology. Microcontroller circuits manufactured in this way have remained functional over 4 years and a water proof and mechanically reliable.
\end{abstract}

\section{Introduction}

Electronics manufacturers face increased legislative pressure to design circuits and systems which are low cost, require minimal energy to produce and recycle, are easily recycled and, if discarded inappropriately in land-fill, cause minimal environmental damage [1-3]. The European Union directives RoHS and WEEE relate to recycling and minimal toxicity $[4,5]$ and the Basel Convention prohibits the international movement of toxic waste so that highly efficient and environmentally benign recycling plants are not available to almost all nations. It is in this legislative environment that the electronics industry has sought alternative manufacturing and recycling technologies.

Design for the Environment (DfE) is a term commonly used to describe design in which the manufacture, use and disposal/recycling of materials of products is oriented for minimal environmental impact. Circuits in Plastic (CiP) [6] is a new technology under consideration by mass manufacturing organizations to address these issues.

Of particular interest is the fact that biodegradable, biocompatible and recycled plastics can be used to form the circuits. The lowest cost and least toxic conductive inks can be used for the circuit connections. The circuit is water-proof and so does not suffer damage due to dust, oxidation, humidity or atmospheric degradation. The plastic can be chosen to be transparent or opaque to optical, radio or magnetic signals for communications and energy harvesting. The inclusion of a passive RFID tag allows the device to be sorted in a solid waste conveyor line for appropriate recycling [7].

When considering DfE, the concept of an energy and toxicity analysis of the product and its manufacture is based on the "cradle to grave" (i.e. from the raw materials to the disposal of the goods into a waste stream) has been replaced with the concept of "cradle to cradle" in which the material recovery and reuse at the end of life is also considered. For example the conversion of vacuum tube televisions (high cost) at their end of life to road base (very low value) is not a desirable "recycling" technology.

This paper will report two major reliability investigations relating to the $\mathrm{CiP}$ manufacturing technique mechanical/electrical connections and thermal properties. The first investigation was experimental and demonstrated that with appropriate ink selection, circuit connectivity was maintained under dog-bone tensile tests. The second investigation was a thermal modeling exercise which demonstrated that the heat lost from a sealed plastic circuit was almost identical to a traditional mounted gull-wing, surface mounted (SMT) circuit.

\section{Circuits in Plastic - Manufacture}

The manufacturing technique for CiP was initially based on a rigid plastic circuit board (injection molded with component pockets on one side and excellent surface finish on outside), standard electronic components or chip level active components are placed in divots or pockets in the board. The board is then screen printed or ink-jet printed with conductive tracks and sealed with a thin sheet of similar plastic. The process avoids copper etching and soldering during manufacture, and the need for an additional enclosure to be manufactured separately is avoided.

The electronic systems created in this way can be made water-proof, the circuits are protected from the environment, the estimated cost of production is estimated to be lower than current techniques, the encapsulation material can include one or more of the following characteristics:
- Optically transparent
- Radio transparent
- Recycled plastic material
- Biodegradable material
- Biocompatible material
- Flexible or rigid circuits

Fig. 1 shows a flashing light circuit constructed using the CiP technique. The circuit consists of two coin cell batteries, 5 surface mount LEDs, a PIC microcontroller, a press button switch and some passive elements. The conductive tracks were screen printed using silver ink. In the laboratory we have demonstrated screen printing to bare silicon die with track widths down to 90 micons. The plastic material was 
polycarbonate sheeting selected for transparency, minimal water uptake and a moderately high, thermal transition temperature $\left(180^{\circ} \mathrm{C}\right)$. The device operates under-water. The circuit is now four years old and has suffered no environmental degradation. This was manufactured in the laboratory as part of a batch of 10 units.

\section{Circuits in Plastic - Disassembly}

There are two basic principles in DfE - minimal energy use, and the reduction of the complexity of the chemical content of the waste streams. The second allows for improved recycling with fewer purification steps and so lower energy requirements. The $\mathrm{CiP}$ manufacturing technique offers a significant reduction in the number of components in the solid waste stream.

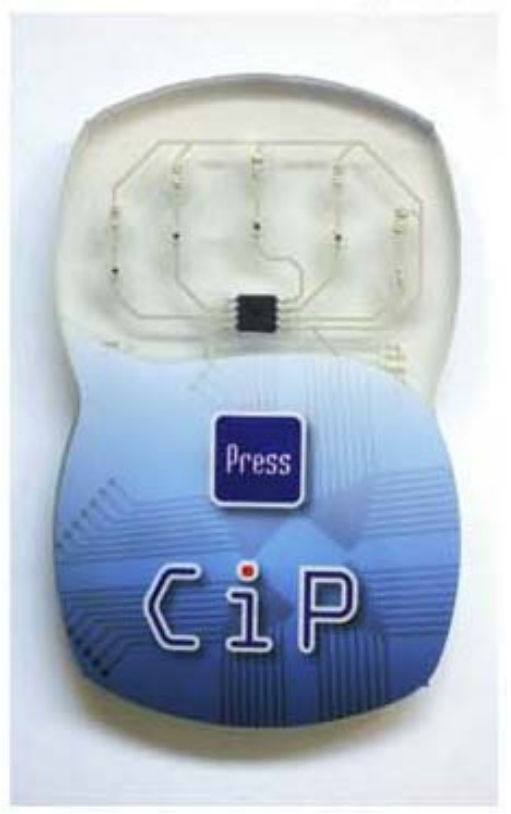

Fig. 1. Flashing light circuit formed using the Circuits in Plastic (CiP) manufacturing technique.

There are two common techniques used around the world for e-waste recycling of circuit boards:

a) The complete product (i.e. including the plastic enclosure and circuit boards) are shredded and then incinerated. The plastic materials and other combustable and volatile components (including aluminum) are expelled from the mix as a vapor. The remaining metals (copper, lead, etc) are separated and purified. The silicon circuits are drawn off in the slag from the furnace and discarded. The complete process is highly automated

b) The circuit boards are removed from the plastic enclosure. The plastic material is either recycled or incinerated. The electronic components are un-soldered from the boards and reused or recycled separately. The bare circuit boards are burnt and the metals recovered. The plastic coated connecting wires in the circuit are burnt to remove the plastic coating and the metals recovered (usually copper and tin/lead solder). The process is usually undertaken by hand in small factories or houses. This has lead to toxicity problems in the local communities.

The strategy for the disassembly for CiP circuits is to mechanically remove the cover sheet. This can be undertaken without additional heat as the thermal bond between the cover sheet and the base is the weakest part of the structure mechanically. The electronic components are removed mechanically for independent recycling or reuse. The conducting tracks on the remaining plastic are removed and recycled and the plastic can be used for new circuit boards. The thermal plastic circuits are disassembled by mechanically removing the cover sheet and then the electronic components allowing recycling of the plastic and more highly concentrated, less complex metal recovery.

\section{Circuits in Plastic - Mechanical Reliability}

In traditional surface mounted printed circuit boards, often the circuit elements are soldered onto one side of the board only. If the circuit board is bent, it is possible that the adhesive holding the copper tracks to the substrate can fail mechanically. In this case the reliability of the circuit is significantly reduced. With the CiP technology, this effect is not present under mild bending conditions. The plastic substrate materials can be selected partly on the basis of Young's modulus and so the circuit can be flexible.

The quality of the electrical contact between the electronic components and the screen printed tracks was the subject of mechanical investigation. The CiP process does not use glue or solder to form electrical connections and relies on the mechanical pressure between the conductive track and the circuit component to complete the circuit. A number of parameters were identified as important characteristics of the conductive material:

Melting point - the ink, once set, needed to withstand the thermal bonding process without significant movement and without cracking.

Ductility - the conductive bridge between the electric components and the tracks needed to remain intake even when there was some stretching of the cover sheet. If the board was made from flexible plastic, slight bends in the circuit board must not result in breaks in the circuit continuity.

The resistance was measured between the ends of $53 \mathrm{~mm} \mathrm{x}$ $2.5 \mathrm{~mm}$ printed silver tracks attached to a dog-bone shaped mechanical element of various materials (polycarbonate, polystyrene, and polyester). The samples were subjected to tensile loading at $1 \mathrm{~mm}$ per minute to an extension of $10 \mathrm{~mm}$. Only the polystyrene did not fail over this extension. Typical raw data is shown in Fig. 2 for single coatings of silver on the three polymers. Note the significant increase in resistance above $4 \mathrm{~mm}$ extensions in both polycarbonate and polystyrene.

Table 1 lists the mean slope of the line in the first $3 \mathrm{~mm}$ of extension. These results indicate that while there are significant changes in the resistance under tensile load, only loads above $4 \mathrm{~mm}(7.5 \%)$ show changes which might lead to circuit malfunction.

$200911^{\text {th }}$ Electronics Packaging Technology Conference 
From these experiments it was evident that the electrical contact between the printed ink and the contact wires did not fail.

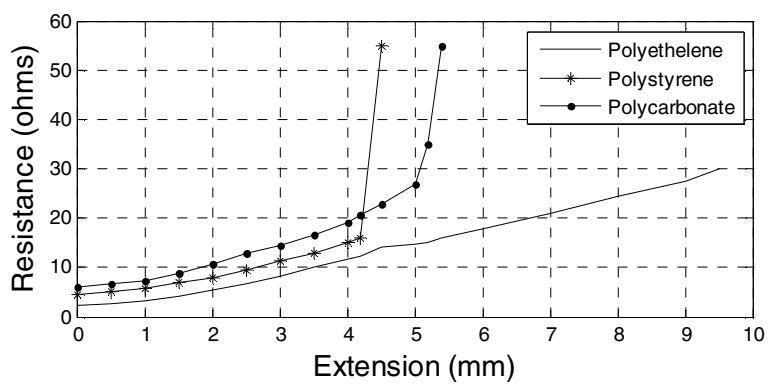

Fig. 2. Resistance variation of single layer, silver printed tracks on polycarbonate, polyester and polystyrene as a function of extension under tensile load.

Table 1. Trend slopes for first $3 \mathrm{~mm}$ extension.

\begin{tabular}{|c|c|}
\hline Polymer Material & Slope $(\Omega / \mathrm{mm})$ \\
\hline Polycarbonate $(1$ coat $)$ & 2.15 \\
\hline Polycarbonate $(2$ coats $)$ & 2.70 \\
\hline Polystyrene $(1$ coat $)$ & 2.74 \\
\hline Polystyrene $(2$ coats) & 2.23 \\
\hline Polyester $(1$ coat) & 0.97 \\
\hline Polyester ( coat) & 0.98 \\
\hline
\end{tabular}

\section{Circuits in Plastic - Thermal Properties}

Most electronic circuits are naturally cooled using convection. In some cases where significant power is dissipated, it is necessary to employ additional measures including forced air movements using a fan, a peltier cooling technique or a heat sink consisting of a fin shaped material of high thermal conductivity. In the latter case, the increased surface area allows for improved heat dissipation using natural convection. In the CiP manufacturing technique, the circuits are completely sealed in plastic preventing direct contact between the air and the circuit element. This is a potential problem that must be addressed.

In plastic encapsulated integrated circuits, the heat dissipation over the active semiconductor material must be relatively uniform as most semiconductor materials are brittle. If the heating is uneven, this can result in a hot spot in the semiconductor and unequal thermal expansion. This can result in the shattering of the semiconductor and circuit failure. Electronic circuit component manufacturers usually include an aluminum base ("flag" and "tie bar') on which the semiconductor is glued. This improves the uniformity of the heat distribution over the semiconductor material to improve reliability. The plastic encapsulation material does not have high thermal conductivity and provides only limited heat sink potential. While standard electronic chip packaging is not designed for heat loss, most active and passive electronics do not require the installation of additional heat dissipation techniques.

A two dimensional thermal model was undertaken using the thermal convention analysis package in COMSOL multiphysics ${ }^{\circledR}$ [8]. This software uses the finite element method to solve for the steady state thermal distribution. A plastic thin quad flatpack 10x10x1mm from Microchip [9] was used as the basis for thermal modeling. This is illustrated in Fig. 3, showing the details of the thermal model with the circuit encapsulated in plastic.

The sir velocity across the surface of the package was set to a relatively slow speed $(2 \mathrm{~m} / \mathrm{sec})$ air flow across the top of the model. The ambient temperature of the air was set at $55^{\circ} \mathrm{C}$ and the die temperature was set at $125^{\circ} \mathrm{C}$ which equates to a chip power consumption of $0.1 \mathrm{~W}$.

The same characteristics were used for a traditional surface mounted device (SMT). The model used is given in Fig. 4.

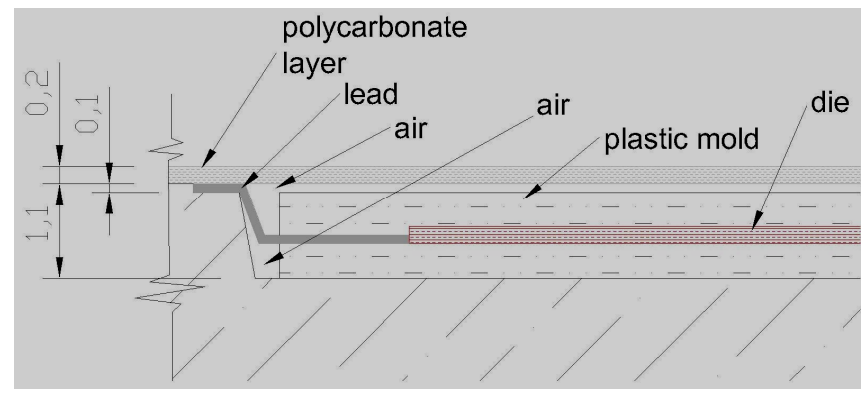

Fig. 3. A section of the model showing the materials of a flat pack circuit encapsulated in polycarbonate using the CiP manufacturing process. The dimensions are in $\mathrm{mm}$.

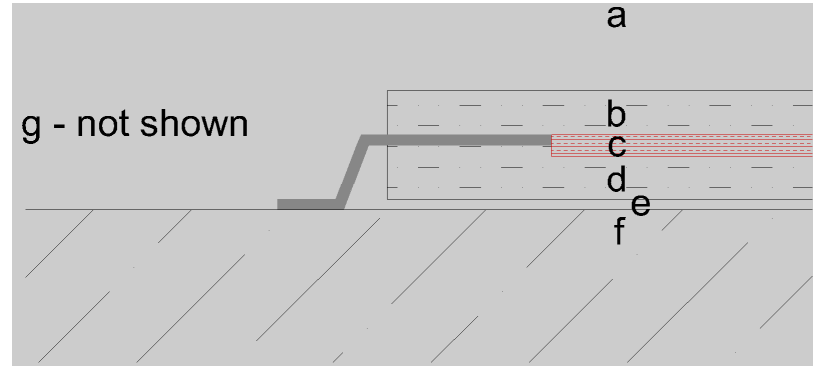

Fig. 4. The model of a traditional SMT: $a$ is air, $b$ is the plastic mould, $\mathrm{c}$ is the semiconductor, $\mathrm{d}$ is the mould, e is an air gap and $f$ is the circuit board material.

The results of the thermal modeling device (the air is flowing left to right) are given in Fig. 5. The steady state temperature distribution for the CiP circuit (top) and the SMT (bottom) shows little temperature difference (less than $5^{\circ} \mathrm{C}$ ) between the silicon die located within the two structures. The highest temperature was found on the leading edge of the SMT.

\section{Discussion and Conclusions}

There are many environmental concerns in the electronics manufacturing industry. In particular, the industry must address the following:

- Energy requirements and the carbon footprint of manufacturing process

- The release of toxic materials during manufacture and recycling

- The recycling of circuits and systems at their end-of-life

- The reduction/elimination of toxic materials and the mix of materials used in manufacture and so the mix of materials in the solid waste stream.

$200911^{\text {th }}$ Electronics Packaging Technology Conference 
The Circuits in Plastic (CiP) manufacturing technique provides a potential solution to all of these issues. In addition, current industry cost estimates for mass manufacture indicate that the cost of production can be substantially reduced through the use of CiP. This comparison is based on lead based soldering and so improved with lead-free soldering manufacture.

Two impediments currently impede the commercial production of electronic circuits and systems using CiP. The first is the lack of a production line to streamline the process for mass manufacture. The second relates to questions of circuit reliability. The reliability has been demonstrated through laboratory prototypes working satisfactorily over a four year period. The results reported in this paper suggest that mechanical and thermal dissipation problems are not an issue. Further work is required to validate these conclusions. The next step is to install a production line for full scale production.

The use of printed conductors poses a cost problem for manufacturers. Currently silver and carbon are used commercially for such electronics as RFID antennas. Silver is relatively expensive and ism also toxic to lower organisms [10]. Recycling is also a problem [11]. Alternative conducting materials are under consideration [12]. In selecting new, printable (screen printing or ink jet technology) an expanded selection criteria should be used. Table 2 highlights the old and additional selection criteria required of these new conductors. A consequence of using new conductors is a decrease in the conductivity of the material and so a decrease in circuit efficiency. In most cases this is not a significant problem as the tracks between circuit elements is very short (less than $10 \mathrm{~mm}$ ) and the line resistance can be decreased by manufacturing processes such as over printing and thermal or UV annealing. The abundance of the elements using in printing conductors is becoming an important cost consideration. The conductors used in electronics and their abundance on earth are given in Table 3. This criteria alone does not regulate price, however, the energy cost of recycling is becoming an important consideration in the electronics industry.

Table 2. Selection criteria for printable conductors.

\begin{tabular}{|c|c|}
\hline Attributes & Properties \\
\hline Effectiveness & Conductivity \\
& Oxidation/Corrosion \\
& Mechanical properties \\
& Patterning techniques \\
& Connections (solder/glue) \\
& Adhesion to substrate \\
& Thermal conductivity \\
& Contact noise \\
\hline Availability & Earth abundance \\
& Refining costs \\
& Recycling costs \\
\hline Toxicity & Solubility in water \\
& Carcinogenicity \\
\hline Energy Costs & Extraction Mining/recycling \\
& Beneficiation/purification \\
& End-of-life recycling \\
\hline
\end{tabular}

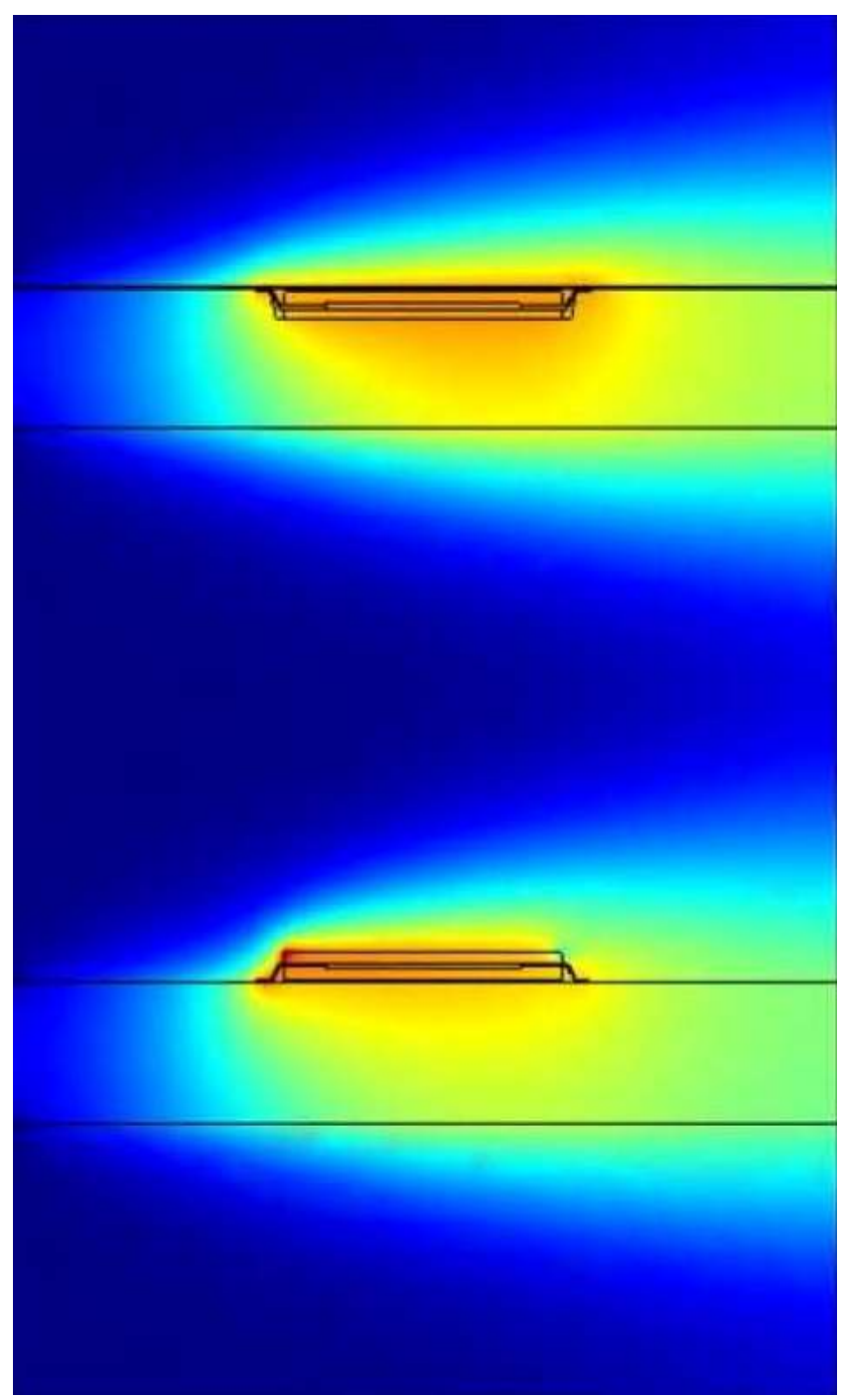

Fig. 5. Thermal temperature distribution of the CiP (top) and SMT (bottom) circuit manufacturing technique.

Table 3. Abundance and toxicity of various printable conductors [10]

\begin{tabular}{|c|c|c|}
\hline Element & $\begin{array}{r}\text { \% Abundance } \\
\text { in Earth's crust }\end{array}$ & Toxicity \\
\hline Aluminum & $\begin{array}{c}8.2 \% \\
\text { Most } \\
\text { abundant metal }\end{array}$ & $\begin{array}{c}\text { Not } \\
\text { dangerous }\end{array}$ \\
\hline Carbon & $480 \mathrm{ppm}$ & Non-toxic \\
\hline Copper & $50 \mathrm{ppm}$ & $\begin{array}{c}\text { Toxic } \\
\text { when taken } \\
\text { in excess }\end{array}$ \\
\hline Silver & $70 \mathrm{ppb}$ & $\begin{array}{c}\text { Toxic to } \\
\text { lower } \\
\text { organisms }\end{array}$ \\
\hline
\end{tabular}

\section{Acknowledgments}

The authors greatly appreciate the work of Gabor Mihala for the thermal modeling and Henning Turnow who undertook the mechanical experimental work. Griffith University has patented the CiP process

$200911^{\text {th }}$ Electronics Packaging Technology Conference 


\section{References}

1. Thiel, D.V., "Sustainable electronics: wireless systems with minimal environmental impact", Proc. International Symposium on Antennas Propagation and EM Theory, Kunming, China, 2008, pp. 1298-1301.

2. Goldberg, L.H. and Middleton, W., (eds) Green electronics, green bottom line: environmentally responsible engineering. Newnes, (Boston ; Oxford , 2000).

3. Stasinopolous, P., Smith, M.H., Hargroves, K., and Desha, C. Whole system design: an integrated approach to sustainable engineering, Earthscan (London, 2009).

4. Europa, "Directive 2002/95/EC of the European Parliament and of the Council of 27 January 2003 on the restriction of the use of certain hazardous substances in electrical and electronic equipment," Official Journal L 03, 13/02/2003 P. $0019-0023$.

5. Europa, "Directive 2002/96/EC of the European Parliament and of the Council of 27 January 2003 on waste electrical and electronic equipment (WEEE) - Joint declaration of the European Parliament, the Council and the Commission relating to Article 9" Official Journal L $037,13 / 02 / 2003$ P. $0024-0039$.

6. Thiel, D.V., and Neeli, M., "Fabrication of electronic circuits in plastic", PCT WO/2007/002995, July 2006.

7. Saar, S., and Thomas, V. "Toward trash that thinks, product tags for environmental management" $J$. Industrial Ecology, vol. 6 (2), pp. 133-146, 2003.

8. COMSOL Multiphysics ${ }^{\circledR}$. "The COMSOL Multiphysics ${ }^{\circledR}$ simulation environment", http://www.comsol.com/products/multiphysics/ accessed September 2009.

9. Microchip Technology Inc, DS61143E-p. 632, www.microchip.com. Accessed September 2009.

10. Emsley, J., Nature's building blocks OUP, (Oxford 2001).

11. Hilliard, H.E., "Silver recycling in the United States in 2000", American Geological survey, Virginia, USA, 2003.

12. Thiel, D.V., and Galehdar, A., "Recent innovations in antenna design for wireless sensors," Proc. International Conference on Electromagnetics in Advanced Applications, Torino, Italy, 2009, pp. 63-65. 